\title{
Which measurements calculated by rotation thromboelastometry were more accurate for platelet counts, Extem-Fibtem or Clotting elasticity?
}

\section{Kodaka M, Ichikawa J, Mori T, Nihei H, Nishiyama K, Komori M. Department of Anaesthesia and Intensive care, Tokyo Women's Medical University Medical Centre East}

Background and Goal of Study

Extem withdrew Fibtem calculated by rotation thromboelastmetry (ROTEM ${ }^{T M}$ ) was reported to be reflect for platelet count ${ }^{1}$. But Solomon et. al. ${ }^{2}$ reported that Extem -Fibtem did not refer to the accurate platelet counts and function. They suggested new parameter, clotting elasticity (CE) calculated by ROTEM value, which could reflect the accurate platelet count and aggregation. The aim of study is to investigate which measurements are more accurate for platelet counts and seek the normal range of CE as well.

\section{Material and Methods}

Patients scheduled cardiac surgery with cardiopulmonary bypass (CPB) were assessed for eligibility. The criteria of transfusion were indicated below, such as hemoglobin $>9 \sim 10 \mathrm{mg} / \mathrm{dl}$, fibrinogen $>150 \sim 200 \mathrm{mg} / \mathrm{dl}$, platelet count $>50,000 \sim 100,000 / \mu \mathrm{l}$. The heparin dose during CPB was administered $300 \sim 400 \mathrm{U} / \mathrm{kg}$ to target activated clotting time (ACT) $>450 \mathrm{sec}$ and reversed by protamine sulfate $60 \sim 100 \%$ dose of heparin. The hemostatic therapy was such as concentrated red cells (CRCs; $140 \mathrm{~g} / \mathrm{unit}$ ), fresh frozen plasma (FFP; 120g/unit), and platelet concentration (PC; i.e.,10unit originated from two liter of blood). We collected the samples in four points, after anesthesia introduction (baseline), just after CPB starting, weaning time from $\mathrm{CPB}$, and the end of surgery.

Results and Discussion:

Seventy patients were enrolled in the study (Table 1: patients characteristics) and we collected 280 points of blood samples to compare the relation between platelet counts and Extem-Fibtem A10 or CE. We also presented the correlation graph between them at figure A and B below. Each correlation coefficient $(R)$ was 0.68 and 0.82 , respectively, which means CE was more accurate than Extem-Fibtem A10. We also found 50 , and 75 of CE (A10) were referred to 50,000 , and $100,000 / \mu l$ of platelet counts, respectively.

CE was a new criterion ${ }^{2}$ and can reflect platelet count and aggregation precisely (Table 2$)$. The calculation formula of CE $=(100 \times E X T E M) /(100-$ EXTEM)- $(100 \times$ FIBTEM)/(100-FIBTEM). But they had not reported how much values were the normal range yet, especially A10.

\begin{tabular}{|c|c|}
\hline Age $(y / o)$ & $69 \pm 9$ \\
\hline Gender (M:F) & $38: 18$ \\
\hline Height $(\mathrm{cm})$ & $161 \pm 8$ \\
\hline Weight（kg） & $59 \pm 11$ \\
\hline ASA score & $3(2-3)$ \\
\hline Euro score II & $2.7 \pm 2.1$ \\
\hline Creatinine $(\mathrm{mg} / \mathrm{dl})$ & $0.9(0.7-1.3)$ \\
\hline \multicolumn{2}{|l|}{ Preoperative complications } \\
\hline Redo surgery & 5 \\
\hline Hypertension & 47 \\
\hline Hyper lipidemia & 14 \\
\hline Arterial fibrillation & 13 \\
\hline Hemolysis dialysis & 5 \\
\hline Diabetes & 15 \\
\hline Respiratory disease & 3 \\
\hline Antiplatelet drugs & 13 \\
\hline Anticoagulants & 14 \\
\hline \multicolumn{2}{|l|}{ Operative methods } \\
\hline Coronary bypass & 2 \\
\hline Single valve repair/replace & 36 \\
\hline Double valve repair/replace & 8 \\
\hline Triple valve repair/replace & 0 \\
\hline Single valve+bypass & 6 \\
\hline Double valve+bypass & 0 \\
\hline Aortic surgery & 4 \\
\hline Anesthesia (min) & $353 \pm 110$ \\
\hline Surgery $(\min )$ & $294 \pm 107$ \\
\hline Cardio-pulmonary bypass $(\mathrm{min})$ & $133 \pm 51$ \\
\hline Crystalloids balance $(\mathrm{ml})$ & $1677 \pm 1056$ \\
\hline Total transfusion balance $(\mathrm{ml})$ & $305 \pm 551$ \\
\hline
\end{tabular}

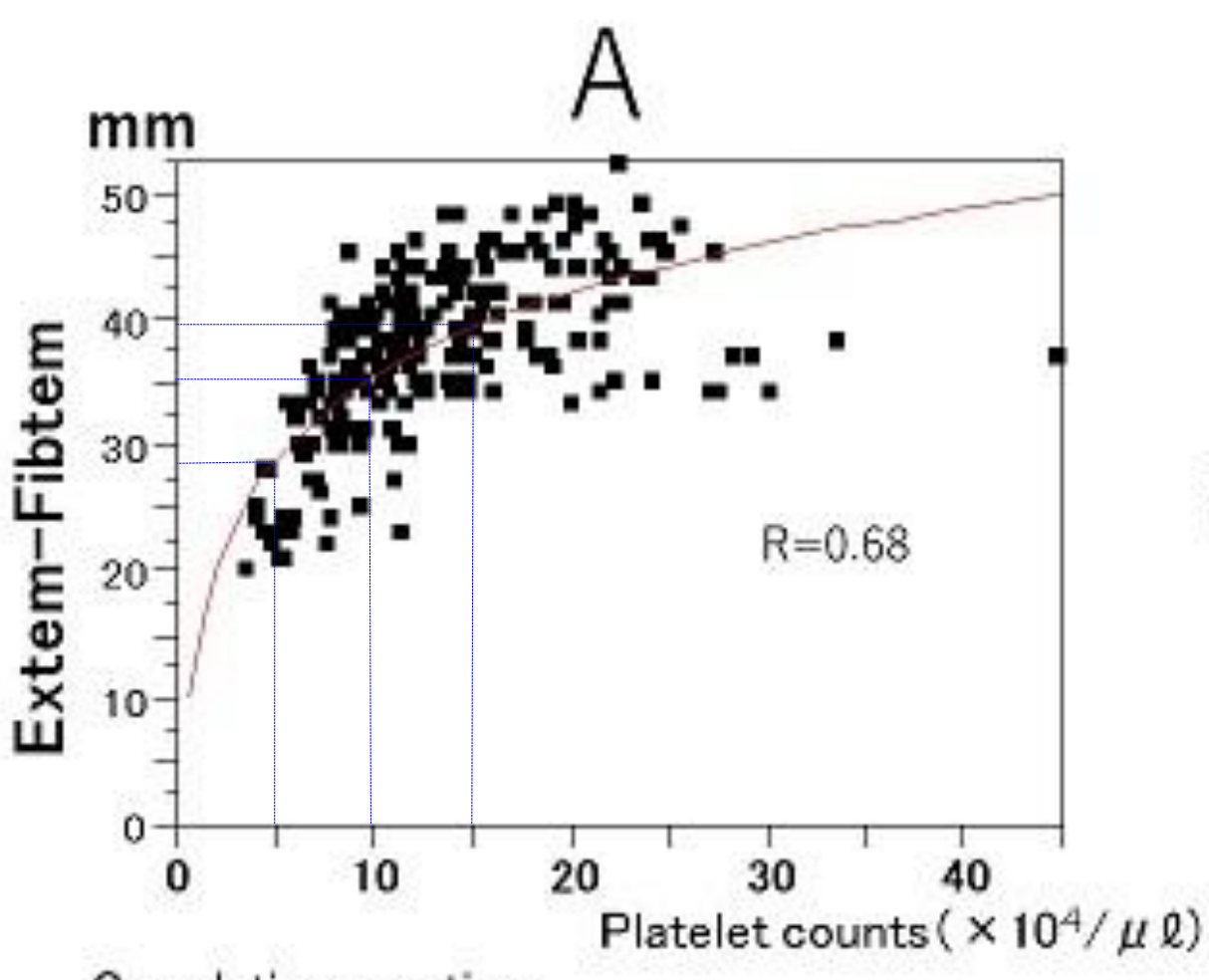

Correlating equation

EXTEM-FIBTEM=13.6 + 9.6 Log Platelet counts

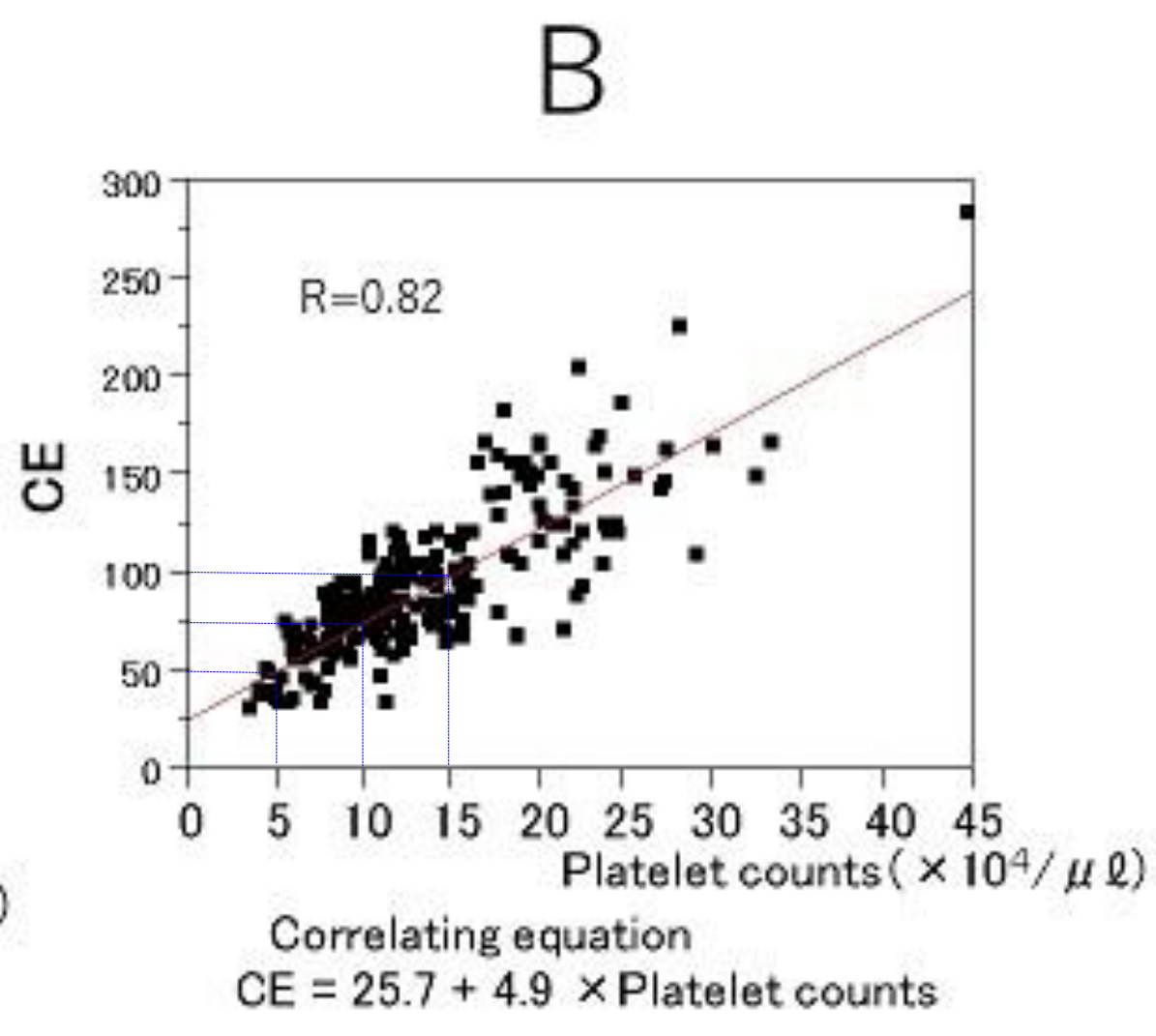

Figure 1 : Correlation between ROTEM ${ }^{\mathrm{TM}}$ values and platelet counts

A. Effective platelet counts (EXTEM A10-FIBTEM A10) and platelet counts

B. Clotting elasticity (CE) and $と$ platelet counts

$\mathrm{R}=$ correlation coefficient

Table 2: Discrepancy between Extem-Fibtem and $\mathrm{CE}^{2)}$

\begin{tabular}{|c|c|c|c|c|c|c|c|c|}
\hline \multirow[b]{2}{*}{$\begin{array}{l}\text { Platelet } \\
\text { count }(/ \mu \mathrm{L})\end{array}$} & \multirow[b]{2}{*}{$\begin{array}{c}\text { FIBTEM } \\
\text { MCF }(\mathrm{mm})\end{array}$} & \multirow[b]{2}{*}{$\begin{array}{c}\text { FIBTEM } \\
\text { MCE }^{a}\end{array}$} & \multirow[b]{2}{*}{$\begin{array}{c}\text { EXTEM } \\
\text { MCF }(\mathrm{mm})\end{array}$} & \multirow[b]{2}{*}{$\begin{array}{l}\text { EXTEM } \\
\text { MCE }^{a}\end{array}$} & \multirow[b]{2}{*}{$\begin{array}{c}\text { MCF } \\
\text { contributors: } \\
\text { fibrin, platelets }\end{array}$} & \multirow[b]{2}{*}{$\begin{array}{c}\text { MCE } \\
\text { contributors: } \\
\text { fibrin, platelets }\end{array}$} & \multirow[b]{2}{*}{$\begin{array}{c}\triangle M C F \text { (EXTEM } \\
\text { - FIBTEM) }\end{array}$} & \multirow{2}{*}{$\begin{array}{c}\text { AMCE (EXTEM } \\
\text { - FIBTEM) } \\
\text { (platelet } \\
\text { component) }\end{array}$} \\
\hline & & & & & & & & \\
\hline 100,000 & 40 & 67 & 70 & 233 & $57.1 \%, 42.9 \%$ & $28.8 \%, 71.2 \%$ & 30 & 167 \\
\hline 50,000 & 20 & 25 & 50 & 100 & $40.0 \%, 60.0 \%$ & $25.0 \%, 75.0 \%$ & 30 & 75 \\
\hline 35,000 & 15 & 18 & 45 & 82 & $33.3 \%, 66.7 \%$ & $22.0 \%, 78.0 \%$ & 30 & 64 \\
\hline 30,000 & 10 & 11 & 40 & 67 & $25.0 \%, 75.0 \%$ & $16.4 \%, 83.6 \%$ & 30 & 56 \\
\hline 20,000 & 5 & 5 & 35 & 54 & $14.3 \%, 85.7 \%$ & $9.3 \%, 90.7 \%$ & 30 & 49 \\
\hline 10,000 & 0 & 0 & 30 & 43 & $0.0 \%, 100.0 \%$ & $0.0 \%, 100.0 \%$ & 30 & 43 \\
\hline
\end{tabular}

${ }^{\mathrm{a}} \mathrm{MCE}=(100 \times \mathrm{MCF}) /(100-\mathrm{MCF})$.

EXTEM $=$ ROTEM ${ }^{*}$ extrinsically activated test; FIBTEM $=$ ROTEM ${ }^{*}$ test designed to assess fibrin-based clotting; $M C E=$ maximum clot elasticity; $M C F=$ maximum clot firmness.

Conclusions:

CE was more accurate than Extem-Fibtem A10. We also detected the border line values of CE (A10), 50 75 were almost corresponded to platelet

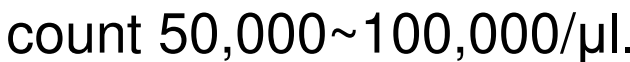

References:

1) Japanese cardiovascular anesthesia 2015; 19: 49-54

2) Anesth Analg 2015;121:868-78 